\title{
NGAL protects in nasopharyngeal carcinoma by inducing apoptosis and blocking epithelial-mesenchymal transition
}

\author{
YING GUO $^{1}$, JIANHUA ZHAI ${ }^{2}$, JING ZHANG $^{1}$ and HUIFANG ZHOU ${ }^{1}$ \\ Departments of ${ }^{1}$ Otorhinolaryngology and ${ }^{2}$ Emergency Internal Medicine, \\ Tianjin Medical University General Hospital, Heping, Tianjin 300052, P.R. China
}

Received July 28, 2019; Accepted December 12, 2019

DOI: $10.3892 / \mathrm{ol} .2020 .11527$

\begin{abstract}
In recent years, neutrophil gelatinase-associated lipocalin (NGAL) has been considered to be a key molecule in different cancer types and its carcinogenesis may be related to the NGAL/MMP-9 complex. However, its expression pattern and role in nasopharyngeal carcinoma (NPC) has rarely been reported. In the current study, 158 tumor tissues from NPC patients were collected and immunohistochemistry was performed to determine the NGAL protein expression, to investigate the correlation between its expression and clinical and pathological parameters using Chi square analysis. Furthermore, by over-expressing NGAL in NPC cell lines, biological alteration of NPC cells with respect to cell proliferation, migration and invasion was analyzed. Results suggested that high expression of NGAL predicts better prognosis and longer survival. Overexpression of NGAL significantly reduced the proliferation and migration of NPC cells, and induced the apoptosis by activating caspase 3,8 and 9, and blocking epithelial-mesenchymal transition by inhibiting mothers against decapentaplegic homolog 2/3 phosphorylation.
\end{abstract}

\section{Introduction}

Nasopharyngeal carcinoma (NPC) is a malignant tumor that has a high incidence in southern China, with an annual incidence rate of nearly 30/100,000 (1). A total of $>70 \%$ of newly diagnosed NPC patients are classified as having locally advanced disease (2). With the advent of concurrent chemotherapy, intensity-modulated radiation therapy (IMRT) and imaging techniques, local control has been significantly improved, and distant metastasis is the main cause of treatment failure in NPC (3). Although some biomarkers were found for evaluating the prognosis of recurrent NPC, the overall survival

Correspondence to: Dr Ying Guo, Department of Otorhinolaryngology, Tianjin Medical University General Hospital, 154 An Shan Road, Heping, Tianjin 300052, P.R. China

E-mail: guoyingtj@163.com

Key words: NGAL, nasopharyngeal carcinoma, epithelialmesenchymal transition, apoptosis, overall survival rate of patients has not improved and the 5-year survival rate is only $30 \%(4,5)$, This makes the treatment of recurrent NPC a major clinical challenge $(6,7)$. Therefore, there is an urgent need to find reliable prognostic markers and effective treatments.

As distant metastasis is a major obstacle for NPC treatment, identifying NPC specific metastasis biomarkers is important for NPC prognosis and predictive treatment. A serum biomarker is the most convenient biomarker for detecting cancer and provides prognostic value for cancer diagnosis, treatment and management. Compared with imaging techniques, serum biomarkers are easier and cheaper for patients $(8,9)$. In the past two decades, neutrophil gelatinase-associated lipocalin (NGAL) has received widespread clinical attention as a biomarker for kidney damage, cardiovascular damage and cancer (10-12). NGAL, also known as lipocalin-2 $(\operatorname{lcn} 2)$, is a $24 \mathrm{kDa}$ glycoprotein in humans encoded by the $1 \mathrm{cn} 2$ gene located at position $3 \mathrm{P} 11$ of chromosome 9 . In recent years, it has become a biomarker for some benign and malignant diseases (13-17). The effect of NGAL in carcinogenesis is dependent on cancer type. Upregulation of NGAL increases cell infiltration in breast, bladder, stomach, gynecological, thyroid, lung, esophageal, colon and chronic myeloid leukemia; but in pancreatic and oral cancer, it reduces cell infiltration $(18,19)$. In addition, upregulation of NGAL can increase the proliferation of cervical cancer and lung cancer cells $(20,21)$. NGAL is a well-known regulatory factor controlling epithelial mesenchymal transition (EMT), invasion and migration. Overexpression of NGAL activates snails, neural-cadherin, fibronectin, matrix metalloproteinase (MMP)-9, nuclear factor- $\varkappa \mathrm{B}$ and other pathways, which in turn upregulates genes involved in stem cells, adhesion, and drug outflow (22-24). Similarly, NGAL silencing reduced migration and invasion by vimentin, MMP-2, and MMP-9, and increased epithelial (E)-cadherin expression (25). These findings suggest that NGAL plays a key role in the development and progression of cancer. Recent studies (26-29) have indicated that NGAL may have pro-oncogenic or anti-oncogenic functions. In fact, its oncogenic effect is related to the complex NGAL/MMP-9; while its anti-tumor effect is related to the inhibition of the pro-neoplastic factor hypoxia inducible factor (HIF)-1a, the HIF-1a-dependent vascular endothelial growth factor and FAK (20). Its role in each cancer type is dependent on the different tumor microenvironment and different signaling pathway activation in cancer types. However, the role of NGAL 
in NPC has not been well confirmed and its expression and role in different stages of development of NPC have not been studied in detail $(30,31)$. Therefore, studying the relationship between the expression of NGAL and the clinical parameters of NPC aids understanding of whether NGAL can be used as a biomarker for the diagnosis and prognosis of NPC.

In this study, the expression of NGAL at different stages of NPC was examined. In addition, through exogenous NGAL transfection, the role of NGAL in the development, proliferation, invasion, migration, EMT and other developmental processes of NPC was investigated.

\section{Materials and methods}

Patients. The present study was approved by the Independent Ethics Committee of the General Hospital of Tianjin Medical University. Before analysis, consent from each patient was received.

In this study, 209 NPC patients were sampled from March 2012 to May 2016 at the Tianjin Medical University General Hospital. Patients were selected according to the following criteria: i) Histologically proven locally advanced NPC with biopsy specimens; ii) Karnofsky score $(>70)$; iii) concurrent chemotherapy based on basic IMRT and cisplatin at the time of initial diagnosis; iv) no malignant tumors or other complications in the past; and v) NGAL staining could be detected in tumor tissues. As a result, 158 patients qualified for this study. All patients were staged using the American Joint Cancer Commission 2010 staging system. The clinical features are listed in Table I. All registered patients received a similar treatment strategy, i.e., IMRT combined with cisplatin-based chemotherapy. Biopsy specimens were obtained by nasal endoscopy for pathological analysis.

Immunohistochemistry (IHC). The expression of NGAL was determined by immunohistochemical analysis. IHC kits (Cell Signaling Technologies, Inc.) were used according to the manufacturer's protocol. Monoclonal antibodies against hNGAL were purchased from Abcam (cat. no. ab23477). Tissue sections were paraffinized and rehydrated with xylene and ethanol, and sealed with 3\% hydrogen peroxide methanol solution for $30 \mathrm{~min}$. After antigen repair, the sections were incubated in a closed solution for $30 \mathrm{~min}$ at $4^{\circ} \mathrm{C}$ and then incubated overnight with the first antibody (1:100 dilution) at $4^{\circ} \mathrm{C}$. The next day, the sections were incubated with the second antibody at room temperature for $1 \mathrm{~h}$ and then stained with $\mathrm{DAB}$ and hematoxylin at room temperature for $10 \mathrm{~min}$ (32).

Scoring. All slides were observed under Nikon Eclipse Ti-E automatic inverted light microscope and the immunoreactivity of NGAL was examined. The staining intensity was graded from 0 to $3+$ (0 for non-staining; $1+$ for weak immunoreactivity; 2+ for moderate immunoreactivity; and 3+ for strong immunoreactivity). Scale numbers 0 and 1 were considered to indicate low expression, while $2+$ and $3+$ were considered to indicate high expression. All the images were captured under x200 magnification.

Cell culture and chemical agents. NPC cancer cell lines C666, HNE-3, CG1 and C666-1 were obtained from the Type
Culture Collection of the Chinese Academy of Sciences. All the cells were cultured in RPMI-1640 (Thermo Fisher Scientific, Inc.) containing 10\% fetal bovine serum (FBS, Gibco; Thermo Fisher Scientific, Inc.) and $100 \mathrm{U} / \mathrm{ml}$ penicillin and $100 \mu \mathrm{g} / \mathrm{ml}$ streptomycin, all cells were cultured in $37^{\circ} \mathrm{C}$ incubator containing $5 \% \mathrm{CO}_{2}$. When growth in logarithmic phase, cells were seeded on 96-well plates for further study.

The mRNA level of NGAL in each cell line was tested by reverse transcription-quantitative (RT-q) PCR. In short, according to the manufacturer's protocol, total RNA was extracted from cells using TRIzol (Thermo Fisher Scientific, Inc.). ReverTra Ace PCR RT kit (Toyobo Life Science) was used to retrieve the RNA (10 $\mu \mathrm{g})$ from each group and obtain the corresponding cDNA at $4^{\circ} \mathrm{C}$. THUNDER RBIRD q-PCR Mix (Toyobo Life Science) was used to carry out RT-PCR on the ABI Prism 7900 Sequence Detection System and Cq was standardized to maintain the signal of GAPDH gene. The fold-change of expression was calculated as $2 \Delta \mathrm{Cq}$ (Treated-Untreated) (33). The primer for GAPDH was 5-AAA CAGAAGGCAGCTTTACGATG-3 and 5-AAATGTTCT GATCCAGTAGCG-3. For NGAL, the sense primer was 5-TC CCAGAGCTGAACGG-3 and anti-sense primer was 5-GAA GTCGCGGAGACA-3. The qPCR cycle conditions were: One cycle of $95^{\circ} \mathrm{C}$ for $30 \mathrm{sec}, 40$ cycles of $95^{\circ} \mathrm{C}$ for $15 \mathrm{sec}, 58^{\circ} \mathrm{C}$ for $30 \mathrm{sec}$ and $72^{\circ} \mathrm{C}$ for $30 \mathrm{sec}$.

Exogenous expression of NGAL. Exogenous expression using PC-DNA3.1-NGAL plasmid vector (cat. no. V79020; Invitrogen; Thermo Fisher Scientific, Inc.) was carried out in the C666 and HNE-3 cell line. Human full length NGAL was cloned and empty vector PCDNA3.1 was also transfected as a control. Briefly, cells were seeded at a concentration of $2.5 \times 10^{4}$ cells /well in $1 \mathrm{ml}$ medium in a 24 -well plate. The next day, cells were transfected with PC-DNA3.1 control and PC-DNA3.1-NGAL plasmids ( $2 \mu \mathrm{g}$ DNA) using Lipofectamine 3000 reagent (Invitrogen; Thermo Fisher Scientific, Inc.). When fresh DMEM (Gibco; Thermo Fisher Scientific, Inc.) was replaced with medium containing transfection reagent, the cells were allowed to recover for $24 \mathrm{~h}$. Then puromycin $(1 \mu \mathrm{g} / \mathrm{ml})$ was used to select cells and establish a stable NGAL transfected clone. Stable transfected cells were used for a proliferation assay, wound healing assay and migration assay. Transient transfected cells were used to analyze apoptosis and EMT.

Cell viability. In short, $2 \times 10^{3}$ cells/well were inoculated into 96-well plates, six replicate wells were incubated for $72 \mathrm{~h}$. After 72 h, $10 \mu 1$ MTT (5 mg/m; cat. no. M2128, Sigma-Aldrich; Merck KGaA) was added to the cells and further cultured. This incubation lasted for $2 \mathrm{~h}$ at $37^{\circ} \mathrm{C}$. The MTT solution was removed and then $100 \mu \mathrm{l}$ DMSO (Merck KGaA) was added to each well. The Infinite M200 Pro (Tecan Group Ltd.) was used to measure absorbance at $570 \mathrm{~nm}$ in $1 \mathrm{~h}$ later (34).

Apoptosis assay by Annexin V-fluorescein isothiocyanate (FITC) and propidium iodide (PI) staining. The apoptosis of C666 and HNE-3 cells was quantified by double staining of Annexin V-PI with FACscan flow cytometry (FACSCanto ${ }^{\mathrm{TM}} \mathrm{II}$; Becton, Dickinson and Company). After $48 \mathrm{~h}$ treatment with $0.5 \%$ bovine serum albumin (Sigma-Aldrich; Merck KGaA) 
Table I. Patient characteristics and significance of neutrophil gelatinase-associated lipocalin expression in clinical parameters.

\begin{tabular}{|c|c|c|c|}
\hline Characteristics & High group $(n=54)$ & Low group $(n=104)$ & P-value \\
\hline Sex & & & 0.738 \\
\hline Male & 29 & 59 & \\
\hline Female & 25 & 45 & \\
\hline Age & & & 0.727 \\
\hline$\geq 50$ & 21 & 38 & \\
\hline$<50$ & 33 & 66 & \\
\hline $\mathrm{BMI}\left(\mathrm{kg} / \mathrm{m}^{2}\right)$ & & & 0.735 \\
\hline$\geq 23$ & 23 & 41 & \\
\hline$<23$ & 31 & 63 & \\
\hline WHO histological type & & & 0.241 \\
\hline Differentiated & 34 & 55 & \\
\hline Undifferentiated & 20 & 49 & \\
\hline EBV infection & & & 0.388 \\
\hline Positive & 32 & 69 & \\
\hline Negative & 22 & 35 & \\
\hline $\mathrm{T}$ classification & & & 0.006 \\
\hline $\mathrm{T} 1+\mathrm{T} 2$ & 30 & 33 & \\
\hline $\mathrm{T} 3+\mathrm{T} 4$ & 24 & 71 & \\
\hline Lymph node metastasis & & & $<0.001$ \\
\hline Absent & 35 & 35 & \\
\hline Present & 19 & 69 & \\
\hline Distant metastasis & & & 0.01 \\
\hline Absent & 23 & 73 & \\
\hline Present & 31 & 31 & \\
\hline Overall stage & & & 0.007 \\
\hline $\mathrm{I}+\mathrm{II}$ & 39 & 29 & \\
\hline III+IV & 15 & 75 & \\
\hline
\end{tabular}

BMI, body mass index; EBV, Epstein-Barr virus.

PBS solution, the harvested cells were washed with cold PBS and then suspended in $200 \mathrm{ml}$ combined buffer $(10 \mathrm{mM}$ HEPES/ $\mathrm{NaOH} \mathrm{pH} 7.4,140 \mathrm{mM} \mathrm{NaCl}$ and $2.5 \mathrm{mM} \mathrm{CaCl}{ }_{2}$ ) and incubated together. A total of $5 \mathrm{ml} \mathrm{Annexin} \mathrm{V-FITC}$ was shielded from light for $10 \mathrm{~min}$ at room temperature. The samples were washed with a buffer solution and re-suspended in PBS. The apoptotic cells were stained with $5 \mu \mathrm{g} / \mathrm{ml}$ PI. The apoptotic cells were identified by flow cytometry using FlowJo 10.0 (FlowJo LLC). The PI has excitation maximum at $535 \mathrm{~nm}$ and fluorescence emission maximum at $617 \mathrm{~nm}$. The vector-transfected cells were used as negative controls. Cells showing Annexin V-/PI+ were considered necrotic, showing that Annexin $\mathrm{V}+/ \mathrm{PI}+$ was considered to be late apoptotic or secondary apoptotic, while Annexin V+/PI-cells were considered to be early or primary apoptotic cells (35).

In vitro wound closure assay. The control PCDNA 3.1 and PCDNA 3.1-NGAL cells were inoculated into 6-well plates and fused, then serum starved for $8 \mathrm{~h}$. the confluent monolayer cells were scraped with the tip of a pipette. PBS was used to wash the plate to remove non-adherent cells and images were captured of the wounds at 0 and $24 \mathrm{~h}$. The edge of the wound was marked and the wound area was measured. Then, the ratio of wound recovery was calculated as follows: Wound recover ratio $=[$ (initial wound area - wound area) / initial wound area] x100\%.

Cell invasion assay. The serum of PCDNA 3.1 and PCDNA 3.1-NGAL cells was starved for $18 \mathrm{~h}$ and then inoculated into a Transwell insert (cat. no. 3422, Corning, Inc.) coated with matrix gel. After starvation, the cells were treated with trypsin and inoculated in the upper chamber of Transwell insert at the concentration of $5 \times 10^{4}$ cells. In the lower chamber, the medium containing $10 \%$ FBS was added as a chemical attractant. Then the cells were incubated at $37^{\circ} \mathrm{C}$ for another $24 \mathrm{~h}$. Migrating cells at the bottom of Transwell insert were fixed in $70 \%$ ethanol for $30 \mathrm{~min}$ at room temperature and stained with crystal violet solution for $30 \mathrm{~min}$ at room temperature. The stained cells were observed under an inverted light microscope (Nikon Eclipse Ti-E) and images were captured with a Nikon 500 camera. After the image was taken, the film was dissolved in 1\% SDS (Sigma-Aldrich; Merck KGaA) solution and the absorbance at $595 \mathrm{~nm}$ was read in Tecan reader at $37^{\circ} \mathrm{C}$ for $1 \mathrm{~h}$. 
Table II. Univariate and multivariate analyses of prognostic parameters for survival in 158 nasopharyngeal carcinoma patients.

\begin{tabular}{|c|c|c|c|c|c|c|}
\hline \multirow[b]{2}{*}{ Prognostic parameters } & \multicolumn{3}{|c|}{ Univariate analysis } & \multicolumn{3}{|c|}{ Multivariate analysis } \\
\hline & HR & $95 \% \mathrm{CI}$ & P-value & HR & $95 \% \mathrm{CI}$ & P-value \\
\hline Expression of NGAL (low vs. high) & 0.278 & $0.184-0.601$ & 0.001 & 0.325 & $0.121-0.601$ & 0.001 \\
\hline Age (years) & 1.587 & $0.872-1.745$ & 0.065 & - & - & - \\
\hline Sex (male vs. female) & 1.255 & $0.723-1.641$ & 0.147 & - & - & - \\
\hline Tumor differentiation & 1.247 & $0.835-1.645$ & 0.122 & 1.356 & $0.656-1.775$ & 0.125 \\
\hline $\mathrm{T}$ classification & 2.435 & $1.557-2.912$ & 0.019 & 2.013 & $0.896-2.145$ & 0.028 \\
\hline Lymphatic metastasis (absent vs. present) & 3.045 & $1.745-4.456$ & 0.005 & 2.885 & $1.224-3.756$ & 0.002 \\
\hline Distant metastasis (absent vs. present) & 4.877 & $2.204-7.011$ & 0.001 & 4.132 & $2.254-5.624$ & 0.001 \\
\hline
\end{tabular}

BMI, body mass index; HR, hazard ratio; CI, confidence interval; NGAL, neutrophil gelatinase-associated lipocalin.

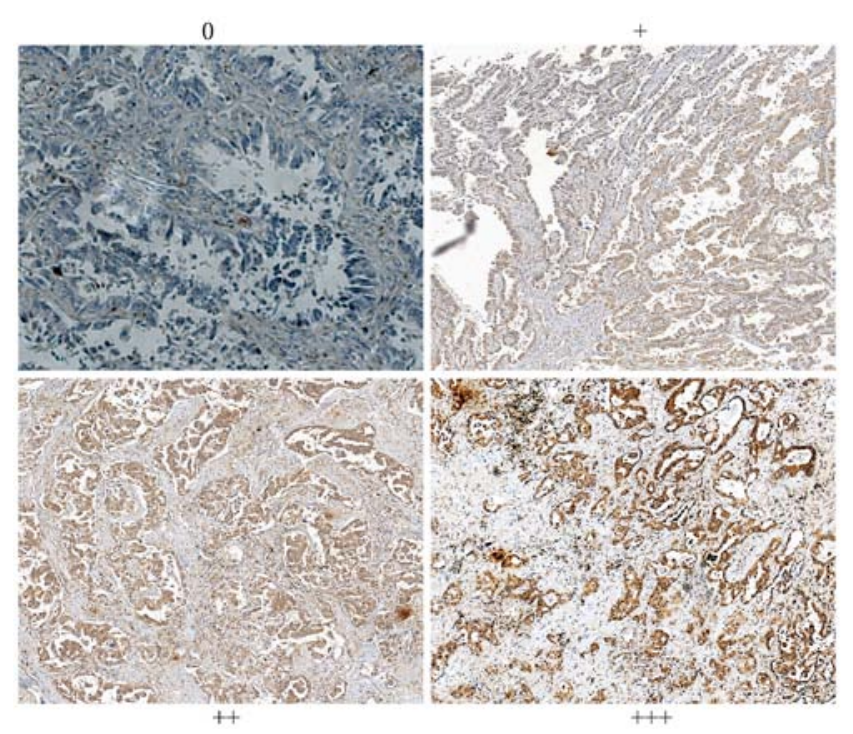

Figure 1. Representative figures of neutrophil gelatinase-associated lipocalin staining in nasopharyngeal carcinoma tumor tissues, scaling is $0,+1,+2$ and +3 . All the pictures were taken at $\times 200$ magnification.

Western blotting. Lysis of NPC cells (HNE-3 and C666) for protein extraction. Protein was extracted with RIPA buffer containing $1 \mathrm{mM}$ PMSF (Sigma-Aldrich; Merck KGaA). The concentration of protein was determined by bicinchoninic acid kit (Pierce; Thermo Fisher Scientific, Inc.). The same amount of protein $(10 \mu \mathrm{g} /$ lane) was carried out by SDS gel electrophoresis (10\%) for $120 \mathrm{~min}$. Protein was transferred to a polyvinylidene fluoride membrane (EMD Millipore). 5\% skimmed milk in Tris buffer saline (TBS) was used to block the membrane at room temperature for $1 \mathrm{~h}$. After blocking, the membrane was incubated overnight at $4^{\circ} \mathrm{C}$ with antibodies, including anti-caspase 3,8 and 9, anti-BCL2, anti-vimentin, anti-smad2/3 and anti-phosphorylated (p)-smad $2 / 3$ and anti- $\beta$-actin. All antibodies were purchased from Abcam. The next morning, the TBS buffer containing $0.05 \%$ Tween-20 (TBST) was used to wash the membrane and the secondary antibody (Abcam; cat. no. ab97040; $1: 3,000)$ linked with horseradish peroxidase was used to detect the membrane for $1 \mathrm{~h}$ at $37^{\circ} \mathrm{C}$. After washing with TBST, the film was developed by enhanced chemilumines-

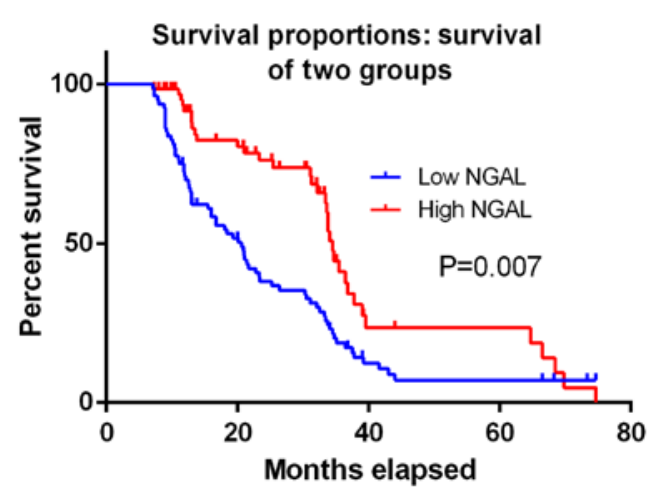

Figure 2. Overall survival curve between NGAL higher group and NGAL lower group by Kaplan-Meier analysis. NGAL, neutrophil gelatinase-associated lipocalin.

cence kit (Yeasen BioTech; cat. no. 36222ES60) and visualized by LAS 4000 imaging system. The intensity of target gene bands normalized relative to the internal control $\beta$-actin was measured and quantified by Image $\mathbf{J}$ software (Version 1.51; National Institute of Health). All reactions were carried out in duplicate and repeated to ensure consistent results (36).

Statistical analysis. Statistical analysis was performed using Graphpad prism 6.0 (GraphPad Software, Inc.). Data were expressed as the mean \pm standard deviation and differences were evaluated by analysis of variance and the Bonferroni post hoc test. Overall survival (OS) was estimated using the Kaplan-Meier method. Univariate analysis was performed using a log-rank test. The exact test of $\chi^{2}$ and Fisher was used to compare the difference between the NGAL high group and the NGAL low group. Multivariate analysis was performed using the Cox proportional hazard model. All statistical tests were bilateral tests. All the experiments were repeated $\geq 3$ times. $\mathrm{P}<0.05$ was considered to indicate a statistically significant difference.

\section{Results}

Patient information. Among the 158 selected patients, 101 were male and 57 were female, with a median age of 52 (ranging 

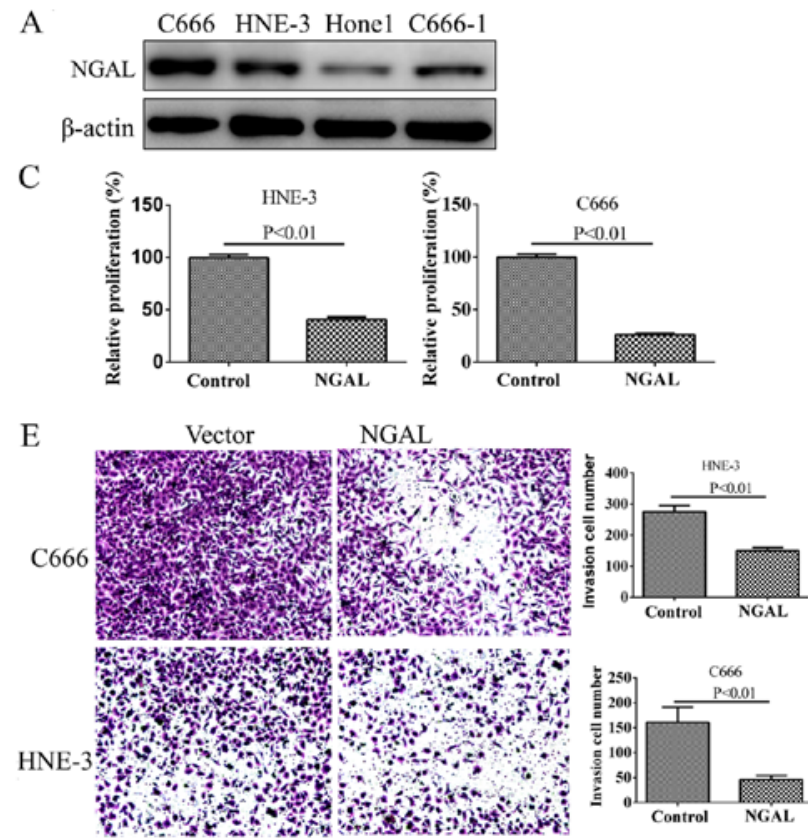
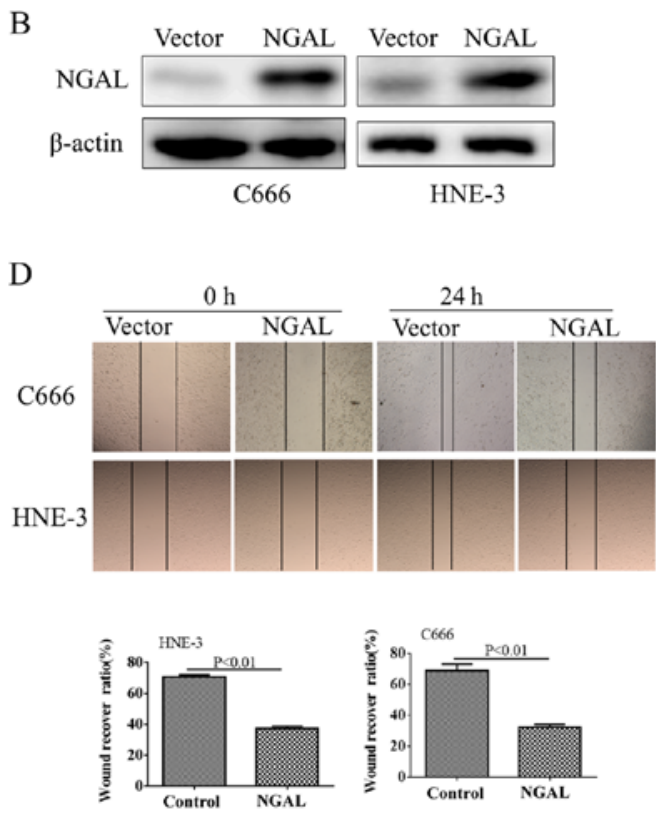

Figure 3. Overexpression of NGAL significantly reduces the NPC cell proliferation, migration and invasion ability. (A) Baseline expression of NGAL in four NPC cell lines. (B) NGAL protein level in stably transfected NGAL and PCDNA-3.1 vector control in C666 cells and HNE-3 cells. (C) An MTT assay was used to test the proliferation capability for stably transfected NGAL and PCDNA-3.1 vector control in C666 cells and HNE-3 cells. (D) Wound healing assay to test the migration capability for stably transfected NGAL and PCDNA-3.1 vector control in C666 cells and HNE-3 cells. (E) Transwell assay to test the invasion capability for stably transfected NGAL and PCDNA-3.1 vector control in C666 cells and HNE-3 cells. Magnification, x40. NGAL, neutrophil gelatinase-associated lipocalin.

from 24 to 77 years). The median body mass index (BMI) was $23.1 \mathrm{~kg} / \mathrm{m}^{2}$ (range, $16.8-32.7 \mathrm{~kg} / \mathrm{m}^{2}$ ). A total of 101 patients were infected with Epstein-Barr virus (EBV; infection rate), the infection rate was $63.9 \%$. All tumors were classified as having a non-keratinized phenotype. After a median follow-up of 60 months, 16 patients $(10.1 \%)$ died and 13 patients $(8.2 \%)$, 9 patients $(5.7 \%)$ and 12 patients $(7.6 \%)$ developed local failure, local failure and distant metastasis, respectively. The 5-year DFS and OS rates were 49.5 and $54.2 \%$, respectively. Detailed patient characteristics are shown in Table I.

Downregulation of NGAL is correlated with poor prognosis of $N P C$. Both cancer cells and lymphocytes can produce NGAL, which is a secreted protein and widely distributed in the whole field of the tumor tissue sections. The representative staining of NGAL in NPC is shown in Fig. 1. Immunohistochemical results showed that all patients were divided into the NGAL low expression group $(\mathrm{N}=104)$ and NGAL high expression group $(\mathrm{N}=54)$, as shown in Table I. NGAL expression was diffuse in tumor tissues. In this study, the expression of NGAL staining was not significantly correlated with age, sex, BMI and EBV infection clinicopathological parameters, but was significantly correlated with clinical stage, lymph metastasis and distant metastasis at diagnosis. Table I summarizes the detailed data, indicating that lower expression is associated with known advanced tumor parameters and metastasis.

The values of various potential prognostic factors were assessed, including age, sex, BMI, overall stage and NGAL predicting OS and DFS. The results of univariate and multivariate analysis are shown in Table II. The present results showed that high NGAL expression was associated with improved OS (5y-OS: 72.4-45.6\%, high to low expression, $\mathrm{P}=0.007$, Fig. 2). In univariate and multivariate analysis, NGAL was considered an independent prognostic factor for OS (the risk ratio of univariate analysis was 0.278 , and that of multivariate analysis was 0.325, all $\mathrm{P}<0.01$ ). Detailed data are shown in Table II.

Exogenous overexpression of NGAL suppresses the proliferation, migration and invasion of NPC cells. The significant downregulation of NGAL expression predicted poor prognosis and survival was observed. The present study therefore proposed to investigate the functional role of NGAL in NPC development and progression. First, the baseline expression of NGAL from four NPC cell lines was tested by western blotting (Fig. 3A). NGAL expression in C666 and HNE-3 was significantly increased compared with the other two cell lines, thus C666 and HNE-3 were selected to for transfection with PC-DNA3.1-NGAL. Stable-transfected clones were selected for MTT, wound healing and transwell assays. Exogenous overexpression of NGAL in C666 and HNE-3 was determined by western blotting (Fig. 3B). From the MTT assay, after growth for $72 \mathrm{~h}$ in contrast with the PCDNA3.1 control, C666-NGAL and HNE-3-NGAL exhibited a significantly decreased proliferation ability (Fig. $3 \mathrm{C} ; \mathrm{P}<0.01$ ). Furthermore, the migration ability and invasion ability of C666-NGAL and HNE-3-NGAL measured by wound healing (Fig. 3D) and transwell assay (Fig. 3E) decreased significantly $(\mathrm{P}<0.01)$, when compared with their corresponding cell lines. These results suggest that NGAL plays a key role in NPC cell growth. Meanwhile, wound healing and migration assays also suggest that overexpression of NGAL could significantly decrease the migration and invasive capability of NPC cells.

Exogenous overexpression of NGAL induces apoptosis of NPC cells by activating caspase family proteins. After a transient transfection for $48 \mathrm{~h}$, the plasmid transfection 

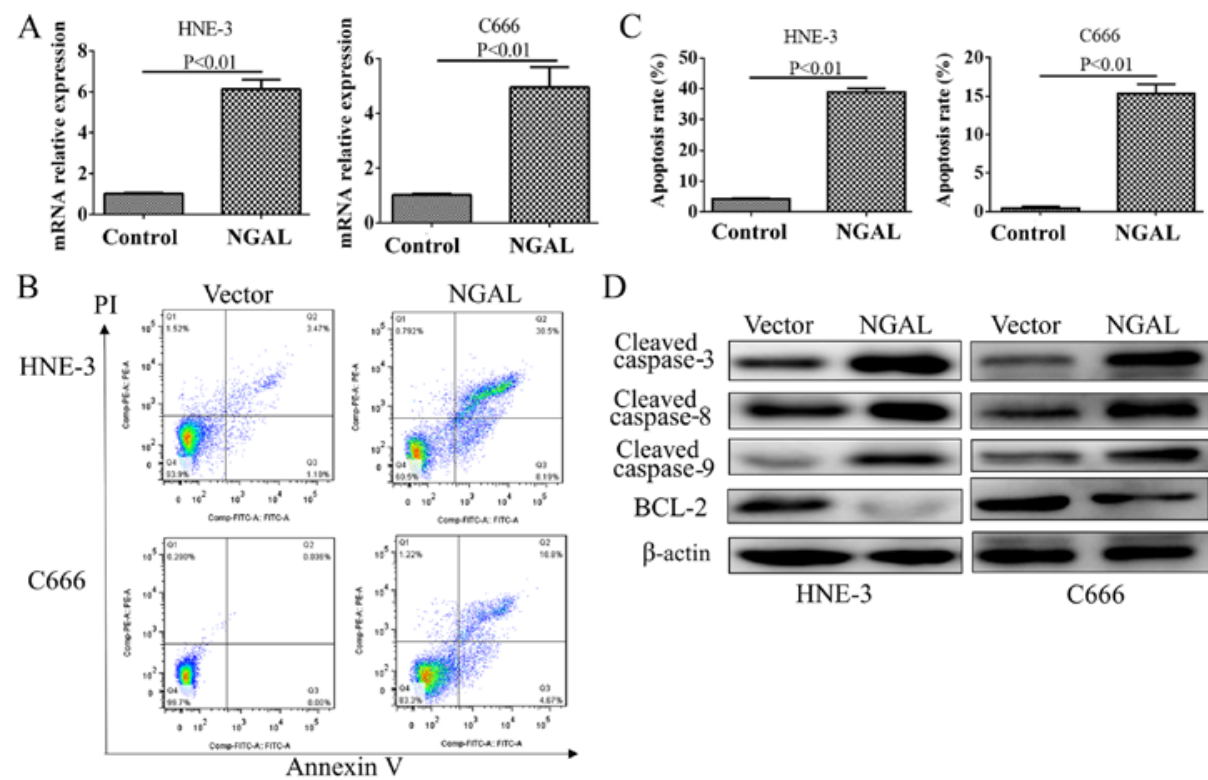

D

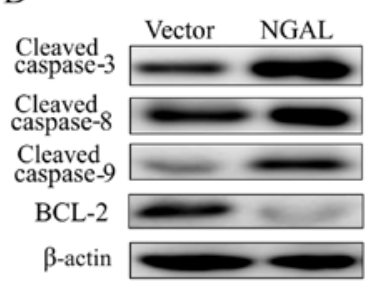

HNE-3

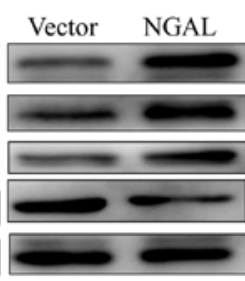

C666

Figure 4. Transient expression of NGAL significantly induces apoptosis in C666 cells and HNE-3 cells. (A) Relative mRNA level of NGAL in transiently transfected NGAL and empty vector control cells by reverse transcription-quantitative PCR after $48 \mathrm{~h}$ transfection. (B) Apoptosis rate examined by flow cytometry. (C) Quantification of apoptosis rate. (D) Caspase members 3, 8 and 9 and BCL-2 was tested by western blotting. NGAL, neutrophil gelatinase-associated lipocalin.

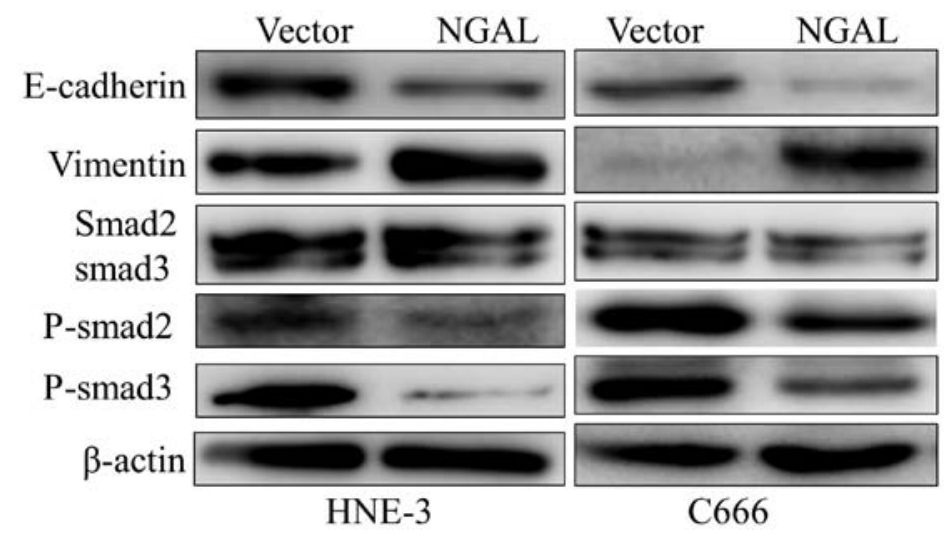

Figure 5. Overexpression NGAL significantly inhibits the epithelial-mesenchymal transition in nasopharyngeal carcinoma cells by blocking the phosphorylation of smad2/3. NGAL, neutrophil gelatinase-associated lipocalin; p-smad, phosphorylated-mothers against decapentaplegic homolog.

efficiency was significantly increased as demonstrated by an RT-qPCR test for NGAL (Fig. 4A). Flow cytometry demonstrated that overexpression of NGAL could produce moderate apoptosis in C666 and HNE-3, which is shown in Fig. 4B and C. By testing caspase family members, cleaved protein of caspase 3,8 and 9 were all found to be decreased in NGAL overexpression cells compared with PC-DNA3.1 vector. However, BCL2 was upregulated by NGAL overexpression in both cell lines (Fig. 4D).

Overexpression of NGAL inhibits the EMT transition in NPC cells. Moreover, the present study found that the cell EMT marker E-cadherin expression was decreased, while vimentin expression was increased when C666 and HNE-3 cells were transfected with PCDNA3.1-NGAL compared with the blank vector groups (Fig. 5). Thus, these results indicated that upregulation of NGAL could suppress the NPC cell EMT process in vitro. Further investigation suggested that overex- pression of NGAL significantly inhibited the $\mathrm{p}-\mathrm{smad} 2 / 3$ and total smad2/3, but inhibition of $p-\operatorname{smad} 2 / 3$ is more effective than total smad $2 / 3$.

\section{Discussion}

There is evidence that NGAL may be a marker of disease status in chronic and acute pathological conditions, especially in inflammatory, metabolic, neurological and cancer diseases (37-39). Multiple studies have explored the possible role of NGAL in various cancer models and have shown that NGAL has beneficial and harmful functions $(37,40)$. Although ongoing research is investigating the value of the NGAL-proMMP-9 complex as an indicator of cancer disease status, there is no detailed data on its full functional significance in disease (41). As a result of reports of the role of NGAL in cancer and the lack of information about NPC, it is necessary to study its role in NPC. 
In the current study, high NGAL was mainly expressed in early stage NPC patients, $72.2 \%$ among patients in stage 1 and stage 2 ; while only $27.8 \%$ of patients from advanced tumors has high expression of NGAL. According to our knowledge, the present study is first time that NPC patients with high NGAL expression have been revealed to have an increased survival outcome, which provides a beneficial biomarker to predict the prognosis of NPC.

The current results also showed that the expression of NGAL in NPC tumors was correlated with improved prognosis of OS and disease-free survival. From the perspective of immunology, previous study demonstrated that NGAL promotes recruitment of tumor infiltrating leukocytes and tumor allografts using wild type thyroid carcinoma cells are decreased compared with tumor allografts from NGAL-depleted cell injected mice (29). In the current study, strong NGAL-immune staining is positively correlated with improved survival and the hypothesis is that NGAL might recruit cytotoxic lymphocytes into NPC tumor tissues to elicit the tumor cells.

Exogenous overexpression of NGAL could inhibit the proliferation, migration and invasiveness in vitro using NPC cells. The NGAL-plasmid was transfected into C666 and HNE-3 successfully. By MTT proliferation, wound healing and transwell assays, it was found that overexpression NGAL in NPC cells inhibited the proliferation and migration of NPC cells. These results suggest NGAL plays a cancer suppressor role in NPC, which is different from most solid tumors $(12,15)$. Overexpression of NGAL also induces moderate apoptosis and further study demonstrated that overexpression of NGAL inhibited the caspase family activation. The present study hypothesized that inducing apoptosis is one of the tumor suppressor mechanisms of NGAL in NPC.

Both the clinical relevance study and the in vitro study demonstrated that higher expression of NGAL could inhibit NPC cell migration and metastasis, and EMT is one of important mechanisms of cancer cell metastasis initiation. In the current study, by western blotting, it was found that overexpression of NGAL blocked EMT and possibly, this is one of mechanisms for NGAL inhibiting the migration and invasiveness of NPC cells. As for the mechanism of inhibiting EMT, the present study demonstrated that NGAL overexpression reduced $\mathrm{p}-\mathrm{smad} 2 / 3$. Solid evidence has suggested that smad2/3 signaling enhancement could induce EMT and inhibiting smad2/3 can reverse EMT $(42,43)$.

There are also several limitations in the current study. Firstly, the patient population is small in the present study, so all the conclusions about NGAL with clinical characteristics need to be further verified using a larger NPC population. Secondly, in the mechanism study, only two cell lines were used in the in vitro study and more NPC cell lines should be used to test what role NGAL plays in different NPC cells, due to different cell lines having various biological backgrounds.

In summary, despite the small population size, the present study demonstrated the evaluation of NGAL expression in NPC tumors for the first time in a Chinese population. Although the population size is small, a significant correlation was observed. A larger population size will be useful to further confirm the prognostic significance of NGAL in NPC. However, lack of investigation of the molecular mechanism is a limitation of this study and the possible molecular regulated targets of NGAL needs further investigation, along with the interfering signaling pathways.

\section{Acknowledgements}

Not applicable.

\section{Funding}

The present study was funded by the project of the Tianjin Health Bureau: Vestibular-auditory function in patients with vestibular migraine (grant no. 2014KZ128).

\section{Availability of data and materials}

The datasets used and/or analyzed during the current study are available from the corresponding author on reasonable request.

\section{Authors' contributions}

YG carried out the laboratory experiments. JHZ conceived the study and participated in its design. $\mathrm{HZ}$ and JZ analyzed data and images. All authors read and approved the final manuscript.

\section{Ethics approval and consent to participate}

The present study was approved by the Independent Ethics Committee of the General Hospital of Tianjin Medical University (Tianjin, China). Prior to analysis, consent from each patient was received.

\section{Patient consent for publication}

All patients agreed the use of their medical data and publication. Informed written consent was provided by all patients.

\section{Competing interests}

The authors declare that they have no competing interests.

\section{References}

1. Lee AW, Ma BB, Ng WT and Chan AT: Management of Nasopharyngeal Carcinoma: Current Practice and Future Perspective. J Clin Oncol 33: 3356-3364, 2015.

2. Mao YP, Xie FY, Liu LZ, Sun Y, Li L, Tang LL, Liao XB, Xu HY, Chen L, Lai SZ, et al: Re-evaluation of 6th edition of AJCC staging system for nasopharyngeal carcinoma and proposed improvement based on magnetic resonance imaging. Int J Radiat Oncol Biol Phys 73: 1326-1334, 2009.

3. Zheng L, Cao C, Cheng G, Hu Q and Chen X: Cytomembranic PD-L1 expression in locoregionally advanced nasopharyngeal carcinoma. OncoTargets Ther 10: 5483-5487, 2017.

4. Hua YJ, Han F, Lu LX, Mai HQ, Guo X, Hong MH, Lu TX and Zhao C: Long-term treatment outcome of recurrent nasopharyngeal carcinoma treated with salvage intensity modulated radiotherapy. Eur J Cancer 48: 3422-3428, 2012.

5. Kong L, Wang L, Shen C, Hu C, Wang L and Lu JJ: Salvage Intensity-Modulated Radiation Therapy (IMRT) for Locally Recurrent Nasopharyngeal Cancer after Definitive IMRT: A Novel Scenario of the Modern Era. Sci Rep 6: 32883, 2016.

6. Chen C, Fee W, Chen J, Chan C, Khong B, Hara W, Goffinet D, Li D and Le QT: Salvage treatment for locally recurrent nasopharyngeal carcinoma (NPC). Am J Clin Oncol 37: 327-331, 2014. 
7. Karam I, Huang SH, McNiven A, Su J, Xu W, Waldron J, Bayley AJ, Kim J, Cho J, Ringash J, et al: Outcomes after reirradiation for recurrent nasopharyngeal carcinoma: North American experience. Head Neck 38 (Suppl 1): E1102-E1109, 2016.

8. Wulfkuhle JD, Liotta LA and Petricoin EF: Proteomic applications for the early detection of cancer. Nat Rev Cancer 3: 267-275, 2003.

9. Dawson S-J, Tsui DWY, Murtaza M, Biggs H, Rueda OM, Chin SF, Dunning MJ, Gale D, Forshew T, Mahler-Araujo B, et al: Analysis of circulating tumor DNA to monitor metastatic breast cancer. N Engl J Med 368: 1199-1209, 2013.

10. Sadar S, Kaspate D and Vyawahare N: Protective effect of L-glutamine against diabetes-induced nephropathy in experimental animal: Role of KIM-1, NGAL, TGF- $\beta 1$, and collagen-1. Ren Fail 38: 1483-1495, 2016.

11. Papadopoulou-Marketou N, Margeli A, Papassotiriou I, Chrousos GP, Kanaka-Gantenbein C and Wahlberg J: NGAL as an Early Predictive Marker of Diabetic Nephropathy in Children and Young Adults with Type 1 Diabetes Mellitus. J Diabetes Res 2017: 7526919, 2017.

12. Liu F, Li N, Yang W, Wang R, Yu J and Wang X: The expression analysis of NGAL and NGALR in clear cell renal cell carcinoma. Gene 676: 269-278, 2018

13. Eilenberg W, Stojkovic S, Kaider A, Piechota-Polanczyk A, Nanobachvili J, Domenig CM, Wojta J, Huk I, Demyanets S and Neumayer C: Neutrophil Gelatinase Associated Lipocalin (NGAL) for Identification of Unstable Plaques in Patients with Asymptomatic Carotid Stenosis. Eur J Vasc Endovasc Surg 57: 768-777, 2019.

14. Sueud T, Hadi NR, Abdulameer R, Jamil DA and Al-Aubaidy HA: Assessing urinary levels of IL-18, NGAL and albumin creatinine ratio in patients with diabetic nephropathy. Diabetes Metab Syndr 13: 564-568, 2019.

15. Oikonomou E, Tsalamandris S, Karlis D, Siasos G, Chrysohoou C, Vogiatzi G, Dimitropoulos S, Charalambous G, Kouskouni E and Tousoulis D: The association among biomarkers of renal and heart function in patients with heart failure: The role of NGAL. Biomarkers Med 12: 1323-1330, 2018.

16. Forster CS, Jackson E, Ma Q, Bennett M, Shah SS and Goldstein SL: Predictive ability of NGAL in identifying urinary tract infection in children with neurogenic bladders. Pediatr Nephrol 33: 1365-1374, 2018.

17. Hunsicker O, Feldheiser A, Weimann A, Liehre D, Sehouli J, Wernecke KD and Spies C: Diagnostic value of plasma NGAL and intraoperative diuresis for AKI after major gynecological surgery in patients treated within an intraoperative goal-directed hemodynamic algorithm: A substudy of a randomized controlled trial. Medicine (Baltimore) 96: e7357, 2017

18. Tang J, Li J, Li S, Li J, Yu C and Wei C: Effect of Inhibiting NGAL Gene Expression on A549 Lung Cancer Cell Migration and Invasion. Zhongguo Fei Ai Za Zhi 18: 187-192, 2015 (In Chinese)

19. Wang PH, Ko JL, Yang SF and Lin LY: Implication of human nonmetastatic clone 23 type 1 and its downstream gene lipocalin 2 in metastasis and patient's survival of cancer of uterine cervix. Int J Cancer 129: 2380-2389, 2011

20. Candido S, Maestro R, Polesel J, Catania A, Maira F, Signorelli SS, McCubrey JA and Libra M: Roles of neutrophil gelatinase-associated lipocalin (NGAL) in human cancer. Oncotarget 5: 1576-1594, 2014.

21. Song B, Zhang H, Jiang L, Chi Y, Tian J, Du W, Yu B and Han Z: Down-regulation of lipocalin 2 suppresses the growth of human lung adenocarcinoma through oxidative stress involving Nrf2/HO-1 signaling. Acta Biochim Biophys Sin (Shanghai) 47: 805-814, 2015

22. Chung IH, Wu TI, Liao CJ, Hu JY, Lin YH, Tai PJ, Lai CH and Lin KH: Overexpression of lipocalin 2 in human cervical cancer enhances tumor invasion. Oncotarget 7: 11113-11126, 2016.

23. Mongre RK, Sodhi SS, Sharma N, Ghosh M, Kim JH, Kim N, Park YH, Shin YG, Kim SJ, Jiao ZJ, et al: Epigenetic induction of epithelial to mesenchymal transition by LCN2 mediates metastasis and tumorigenesis, which is abrogated by NF- $x \mathrm{~B}$ inhibitor BRM270 in a xenograft model of lung adenocarcinoma. Int J Oncol 48: 84-98, 2016.

24. Leung L, Radulovich N, Zhu CQ, Organ S, Bandarchi B, Pintilie M, To C, Panchal D and Tsao MS: Lipocalin2 promotes invasion, tumorigenicity and gemcitabine resistance in pancreatic ductal adenocarcinoma. PLoS One 7: e46677, 2012.

25. Monisha J, Roy NK, Padmavathi G, Banik K, Bordoloi D Khwairakpam AM, Arfuso F, Chinnathambi A, Alahmadi TA, Alharbi SA, et al: NGAL is downregulated in oral squamous cell carcinoma and leads to increased survival, proliferation, migration and chemoresistance. Cancers (Basel) 10: 228, 2018.
26. Dertli R, Biyik M, Yolacan R, Karakarcayildiz A, Keskin M, Kayar Y and Asil M: May Neutrophil Gelatinase-Associated Lipocalin (NGAL) Level Predict Mortality in Patients with Hepatocellular Carcinoma (HCC)? J Gastrointest Cancer: Nov 15, 2019 (Epub ahead of print).

27. Li H, Xu Q, Wang Y, Chen K and Li J: Serum neutrophil gelatinase associated lipocalin (NGAL) as a biomarker for predicting high dose methotrexate associated acute kidney injury in children with acute lymphoblastic leukemia. Cancer Chemother Pharmacol 85: 95-103, 2020.

28. Han MY, Nie JW, Li YY, Zhu YZ and Wu G: Downregulation of NGAL is required for the inhibition of proliferation and the promotion of apoptosis of human gastric cancer MGC-803 cells. Cell Physiol Biochem 50: 694-705, 2018.

29. Pacifico F, Pisa L, Mellone S, Cillo M, Lepore A and Leonardi A NGAL promotes recruitment of tumor infiltrating leukocytes. Oncotarget 9: 30761-30772, 2018.

30. Hiromoto T, Noguchi K, Yamamura M, Zushi Y, Segawa E, Takaoka K, Moridera K, Kishimoto $\mathrm{H}$ and Urade $\mathrm{M}$ : Up-regulation of neutrophil gelatinase-associated lipocalin in oral squamous cell carcinoma: Relation to cell differentiation. Oncol Rep 26: 1415-1421, 2011.

31. Lin CW, Yang WE, Lee WJ, Hua KT, Hsieh FK, Hsiao M, Chen CC, Chow JM, Chen MK, Yang SF, et al: Lipocalin 2 prevents oral cancer metastasis through carbonic anhydrase IX inhibition and is associated with favourable prognosis. Carcinogenesis 37: 712-722, 2016.

32. Zhang S, Xin H, Li Y, Zhang D, Shi J, Yang J and Chen X: Skimmin, a Coumarin from Hydrangea paniculata, Slows down the Progression of Membranous Glomerulonephritis by Anti-Inflammatory Effects and Inhibiting Immune Complex Deposition. Evid Based Complement Alternat Med 2013: 819296, 2013.

33. Livak KJ and Schmittgen TD: Analysis of relative gene expression data using real-time quantitative PCR and the 2(-Delta Delta C(T)) method. Methods 25: 402-408, 2001.

34. Sen Z, Zhan XK, Jing J, Yi Z and Wanqi Z: Chemosensitizing activities of cyclotides from Clitoria ternatea in paclitaxel-resistant lung cancer cells. Oncol Lett 5: 641-644, 2013.

35. Zhang S, Fu Y, Wang D and Wang J: Icotinib enhances lung cancer cell radiosensitivity in vitro and in vivo by inhibiting MAPK/ERK and AKT activation. Clin Exp Pharmacol Physiol 45: 969-977, 2018.

36. Zhang S, Yang J, Li H, Li Y, Liu Y, Zhang D, Zhang F, Zhou W and Chen X: Skimmin, a coumarin, suppresses the streptozotocin-induced diabetic nephropathy in wistar rats. Eur J Pharmacol 692: 78-83, 2012.

37. Xiao X, Yeoh BS and Vijay-Kumar M: Lipocalin 2: An Emerging Player in Iron Homeostasis and Inflammation. Annu Rev Nutr 37: 103-130, 2017.

38. Lippi G, Meschi T, Nouvenne A, Mattiuzzi C and Borghi L: Neutrophil gelatinase-associated lipocalin in cancer. Adv Clin Chem 64: 179-219, 2014.

39. Bauvois B and Susin SA: Revisiting Neutrophil Gelatinase Associated Lipocalin (NGAL) in Cancer: Saint or Sinner? Cancers (Basel) 102018

40. Chakraborty S, Kaur S, Guha S and Batra SK: The multifaceted roles of neutrophil gelatinase associated lipocalin (NGAL) in inflammation and cancer. Biochim Biophys Acta 1826: 129-169, 2012.

41. Bouchet S and Bauvois B: Neutrophil Gelatinase-Associated Lipocalin (NGAL), Pro-Matrix Metalloproteinase-9 (pro-MMP-9) and Their Complex Pro-MMP-9/NGAL in Leukaemias. Cancers (Basel) 6: 796-812, 2014.

42. Valcourt U, Kowanetz M, Niimi H, Heldin CH and Moustakas A TGF- $\beta$ and the Smad signaling pathway support transcriptomic reprogramming during epithelial-mesenchymal cell transition. Mol Biol Cell 16: 1987-2002, 2005.

43. Vincent T, Neve EP, Johnson JR, Kukalev A, Rojo F, Albanell J, Pietras K, Virtanen I, Philipson L, Leopold PL, et al: A SNAIL1-SMAD3/4 transcriptional repressor complex promotes TGF- $\beta$ mediated epithelial-mesenchymal transition. Nat Cell Biol 11: 943-950, 2009.

This work is licensed under a Creative Commons Attribution-NonCommercial-NoDerivatives 4.0 International (CC BY-NC-ND 4.0) License. 